\title{
Approximate Solutions to the Forced Damped Parametric Driven Pendulum Oscillator: Chebyshev Collocation Numerical Solution
}

\section{Wedad Albalawi}

Princess Nourah Bint Abdulrahman University

Alvaro H. Salas

Universidad Nacional de Colombia

S. A. El-Tantawy ( $\nabla$ samireltantawy@yahoo.com )

Port Said University

\section{Research Article}

Keywords: forced damped parametric driven pendulum oscillator, Chebyshev collocation , numerical solution

Posted Date: September 29th, 2021

DOI: https://doi.org/10.21203/rs.3.rs-929150/v1

License: (9) This work is licensed under a Creative Commons Attribution 4.0 International License. Read Full License 


\title{
Approximate solutions to the forced damped parametric driven pendulum oscillator: Chebyshev collocation numerical solution
}

\author{
Wedad Albalawi ${ }^{1, *}$, Alvaro H. Salas ${ }^{2}$, and S. A. El-Tantawy $y^{3,4, *, a}$ \\ ${ }^{1}$ Department of Mathematical Sciences, College of Science, \\ Princess Nourah Bint Abdulrahman University, Riyadh 11656, Saudi Arabia. \\ ${ }^{2}$ Department of Mathematics and Statistics, Universidad Nacional de Colombia, FIZMAKO Research Group, Colombia. \\ ${ }^{3}$ Department of Physics, Faculty of Science, Port Said University, Port Said 42521, Egypt. and \\ ${ }^{4}$ Research Center for Physics (RCP), Department of Physics, \\ Faculty of Science and Arts, Al-Mikhwah, Al-Baha University, Saudi Arabia.
}

(Dated: 23 September 2021)

\begin{abstract}
In this work, novel semi-analytical and numerical solutions to the forced damped driven nonlinear $($ FDDN) pendulum equation on the pivot vertically for arbitrary angles are obtained for the first time. The semi-analytical solution is derived in terms of the Jacobi elliptic functions with arbitrary elliptic modulus. For the numerical analysis, the Chebyshev collocation numerical method is introduced for analyzing the forced damped parametric driven pendulum equation. Moreover, the semi-analytical solution and Chebyshev collocation numerical solution are compared with the Runge-Kutta (RK) numerical solution. Also, the maximum distance error to the obtained approximate solutions is estimated with respect to the RK numerical solution. The obtained results help many authors to understand the mechanism of many phenomena related to the plasma physics, classical mechanics, quantum mechanics, optical fiber, and electronic circuits.
\end{abstract}

* Corresponding author

a) samireltantawy@yahoo.com

\section{INTRODUCTION}

The pendulum oscillator and some related equation have been used as a physical model to solve several natural problems related to bifurcations, oscillations, and chaos such as nonlinear plasma oscillations [1-5], Duffing oscillators [6-10], Helmholtz oscillations [8], rigid plates that satisfy the Johanessen performance criteria [11], transverse vibrations nonlinear of a plate carrying a concentrated mass [12], a beam supported by a double periodic axial oscillating mount [13], cracks subjected to concentrated forces [14], surface waves in a plasma column [15], coupled modes of nonlinear bending vibrations of a circular ring [16], double spin spacecraft [17], motion of spacecraft over slowly rotating asteroids [18], nonlinear vibration of clasped beams [19], the nonlinear equation of wave [20], nonlinear mathematical models of DNA [21], the nonlinear Schrödinger equation etc. There are few attempts for analyzing the equation of motion of the nonlinear damped pendulum taking the friction forces into account [22]. The approximate solution was obtained in form of the Jacobi elliptic functions. However, there are many others forces in addition to the friction force that affect on the motion of the pendulum such as perturbed and periodic forces. These forces appear in different dynamic systems and cannot be neglected due to their great impact on the behavior of the oscillator. For instance, the unforced damped driven nonlinear pendulum equation/or the unforced damped parametric driven pendulum equation

$$
\ddot{\varphi}+2 \beta \dot{\varphi}+\phi(t) \sin \varphi=0,
$$

has been derived in details in Ref. [23], where $\phi(t)=$ $\omega_{0}^{2}-\varepsilon \omega_{2} \cos (\gamma t), \omega_{0}^{2}=g / l, \beta=\mu / 2 m l, \omega_{1}=\gamma / m l$, $\omega_{2}=\gamma^{2} / l, \varepsilon<<1$ is a small parameter, and $\varphi \equiv \varphi(t)$ denotes the angular displacement. In Eq. (1), $\omega_{0}$ indicates the eigenfrequency of the system and $\beta$ represents the damping coefficient. Here, the pendulum is modeled by a sphere of mass $m$, hanging at the end of a massless wire with length $l$ and fixed to a supporting point "O", swinging to and from in a vertical plane under the gravity acceleration " $g$ ". For $\left(\beta, \omega_{2}, F\right)=(0,0,0)$, the unforced undamped nonlinear pendulum oscillation/ the unforced undamped Duffing oscillator is recovered [5]. Equation (1) has only been analyzed numerically via the midpoint scheme and based on our comprehensive survey, we did not find any attempt to find a semi-analytical solution to this equation. Furthermore and motivated by the potential applications of the nonlinear oscillators, the forced damped driven nonlinear (FDDN) pendulum equation or sometimes is called the forced damped parametric driven pendulum equation will be studied

$$
\ddot{\varphi}+2 \beta \dot{\varphi}+\phi(t) \sin \varphi=F \cos (\Omega t),
$$

Also, some approximate analytical solutions to Eq. (2) and some related equations will be obtained for the first time and will compare with the Runge-Kutta (RK) numerical solution. Moreover, the Chebyshev collocation numerical method is introduced for analyzing both Eqs. (1) and (2). Also, we make a comparison between the approximate analytical and numerical solutions by estimating the maximum distance errors. 


\section{A SEMI-ANALYTICAL SOLUTION TO THE FDDN PENDULUM EQUATION}

Let us now writing the evolution equation in the form of the initial value problem (i.v.p.)

$$
\left\{\begin{array}{c}
\ddot{\varphi}+2 \beta \dot{\varphi}+\phi(t) \sin \varphi=F \cos (\Omega t) \\
\varphi(0)=\varphi_{0} \text { and } \varphi^{\prime}(0)=\dot{\varphi}_{0}
\end{array}\right.
$$

where $\varphi(t=0)=\varphi_{0}$ indicates the oscillation amplitude.

Since $\sin \varphi \approx \varphi-2 / 13 \varphi^{3}$, the forced damped parametric driven pendulum i.v.p. (3) could be reduced to the variable-coefficients forced damped Duffing i.v.p.

$$
\left\{\begin{array}{c}
\mathbb{Q} \equiv \ddot{\varphi}+2 \beta \dot{\varphi}+\phi(t)\left(\varphi-\frac{2}{13} \varphi^{3}\right)-F \cos (\Omega t)=0 \\
\varphi(0)=\varphi_{0} \text { and } \varphi^{\prime}(0)=\dot{\varphi}_{0}
\end{array}\right.
$$

Suppose the solution of this problem is given by

$$
\left\{\begin{array}{c}
\varphi=\theta+c_{1} \cos (\Omega t)+c_{2} \sin (\Omega t) \\
\theta(0) \equiv \theta_{0}=\varphi_{0}-c_{1} \text { and } \varphi^{\prime}(0) \equiv \theta_{1}=\dot{\varphi}_{0}-c_{2} \Omega
\end{array}\right.
$$

The function $\theta \equiv \theta(t)$ is a solution to the below ODE

$$
\ddot{\theta}+2 \beta \dot{\theta}+\phi(t)\left(\theta-\frac{2}{13} \theta^{3}\right)=0 .
$$

Accordingly, we get

$$
\begin{aligned}
& \mathbb{Q}=-\frac{1}{26} \cos (t \Omega)\left(\begin{array}{c}
\phi(t)\left(3 c_{1}^{3}+3 c_{2}^{2} c_{1}-26 c_{1}\right) \\
-52 c_{2} \beta \Omega+26 c_{1} \Omega^{2}+26 F
\end{array}\right) \\
& -\frac{1}{26} \sin (t \Omega)\left(\begin{array}{c}
\phi(t)\left(3 c_{2}^{3}+3 c_{1}^{2} c_{2}-26 c_{2}\right) \\
+\left(52 c_{1} \beta \Omega+26 c_{2} \Omega^{2}\right)
\end{array}\right) \\
& -\frac{3}{13} \theta \phi(t)\left(\begin{array}{c}
\left(c_{1}^{2}-c_{2}^{2}\right) \cos (2 t \Omega) \\
+c_{1}^{2}+c_{2}^{2}+2 c_{2} c_{1} \sin (2 t \Omega)
\end{array}\right) \\
& -\frac{6}{13} \theta^{2} \phi(t)\left(c_{2} \sin (t \Omega)+c_{1} \cos (t \Omega)\right) \\
& -\frac{\phi(t)}{26}\left[c_{2}\left(3 c_{1}^{2}-c_{2}^{2}\right) \sin (3 t \Omega)+c_{1}\left(c_{1}^{2}-3 c_{2}^{2}\right) \cos (3 t \Omega)\right] .
\end{aligned}
$$

Now, for small $\gamma$, we get $\phi(t)=\omega_{0}^{2}-\varepsilon \omega_{2} \cos (\gamma t) \approx \omega_{0}^{2}-$ $\varepsilon \omega_{2}=\kappa$ which leads to

$$
\begin{aligned}
& \mathbb{Q} \approx-\frac{1}{26} \cos (t \Omega)\left(\begin{array}{c}
3 c_{1}^{3} \kappa+3 c_{2}^{2} c_{1} \kappa-26 c_{1} \kappa \\
-52 c_{2} \beta \Omega+26 c_{1} \Omega^{2}+26 F
\end{array}\right) \\
& -\frac{1}{26} \sin (t \Omega)\left(\begin{array}{c}
3 c_{2}^{3} \kappa+3 c_{1}^{2} c_{2} \kappa-26 c_{2} \kappa \\
+52 c_{1} \beta \Omega+26 c_{2} \Omega^{2}
\end{array}\right) \\
& -\frac{3}{13} \theta \phi(t)\left(\begin{array}{c}
2 c_{2} c_{1} \sin (2 t \Omega)+c_{1}^{2} \cos (2 t \Omega) \\
-c_{2}^{2} \cos (2 t \Omega)+c_{1}^{2}+c_{2}^{2}
\end{array}\right) \\
& -\frac{6}{13} \theta^{2} \phi(t)\left(c_{2} \sin (t \Omega)+c_{1} \cos (t \Omega)\right) \\
& -\frac{\phi(t)}{26}\left[c_{2}\left(3 c_{1}^{2}-c_{2}^{2}\right) \sin (3 t \Omega)+c_{1}\left(c_{1}^{2}-3 c_{2}^{2}\right) \cos (3 t \Omega)\right] .
\end{aligned}
$$

The constants $c_{1}$ and $c_{2}$ could be determined from the below system

$$
3 c_{1}^{3} \kappa+3 c_{2}^{2} c_{1} \kappa-26 c_{1} \kappa-52 c_{2} \beta \Omega+26 c_{1} \Omega^{2}+26 F=0,
$$$$
3 c_{2}^{3} \kappa+3 c_{1}^{2} c_{2} \kappa-26 c_{2} \kappa+52 c_{1} \beta \Omega+26 c_{2} \Omega^{2}=0 \text {. }
$$

Eliminating $c_{2}$ from the system (9) gives the below cubic equation

$$
\begin{aligned}
& -26 c_{1}\left(\begin{array}{c}
3 F^{2} \kappa^{2}-3 F^{2} \kappa \Omega^{2}-416 \beta^{4} \Omega^{4} \\
-104 \beta^{2} \kappa^{2} \Omega^{2}+208 \beta^{2} \kappa \Omega^{4}-104 \beta^{2} \Omega^{6}
\end{array}\right)+9 F^{2} \kappa^{2} c_{1}^{3} \\
& -624 F \beta^{2} \kappa \Omega^{2} c_{1}^{2}+78 F^{3} \kappa-2704 F \beta^{2} \kappa \Omega^{2}+2704 F \beta^{2} \Omega^{4}=0 .
\end{aligned}
$$

Eliminating $c_{1}$ from the system (9) gives the following cubic equation

$$
\begin{aligned}
& \left(10816 \beta^{4} \Omega^{4}+2704 \beta^{2} \kappa^{2} \Omega^{2}-5408 \beta^{2} \kappa \Omega^{4}+2704 \beta^{2} \Omega^{6}\right) c_{2} \\
& +9 F^{2} \kappa^{2} c_{2}^{3}+\left(312 F \beta \kappa \Omega^{3}-312 F \beta \kappa^{2} \Omega\right) c_{2}^{2}-5408 F \beta^{3} \Omega^{3}=0 .
\end{aligned}
$$

We choose the least in magnitude pair of real roots $\left(c_{1}, c_{2}\right)$ in Eqs. (10) and (11). Accordingly, the final form of the approximate analytical solution to the i.v.p. (3) is given by

$$
\varphi_{\text {approx }}(t)=\theta(t)+c_{1} \cos (t \Omega)+c_{2} \sin (t \Omega),
$$

with

$$
\begin{aligned}
& \theta(t)=\frac{e^{\beta(-t)}\left(\begin{array}{c}
b_{1} \operatorname{dn}(f(t) \sqrt{\omega} \mid m) \operatorname{sn}(f(t) \sqrt{\omega} \mid m) \\
+\theta_{0} \operatorname{cn}(f(t) \sqrt{\omega} \mid m)
\end{array}\right)}{1+b_{2} \operatorname{sn}(f(t) \sqrt{\omega} \mid m)^{2}} \\
& f(t)=\frac{2 \sqrt{\frac{-330 \beta^{2}-329 \kappa}{\kappa}}}{\sqrt{329} \gamma} E\left(\frac{t \gamma}{2} \mid \frac{658 \varepsilon \omega_{2}}{330 \beta^{2}-329 \kappa}\right),
\end{aligned}
$$

where

$$
\begin{gathered}
\omega=-\frac{p}{2 m-1}, m=\frac{1}{2}\left(1-\frac{p}{\sqrt{\left(p+q \varphi_{0}^{2}\right)^{2}+2 \theta_{1}^{2} q}}\right), \\
b_{1}=\frac{\delta \theta_{1} \sqrt{1-2 m}}{\sqrt{p}}, b_{2}=\frac{p+q \varphi_{0}^{2}-\omega}{2 \omega}, p=\kappa, q=-\frac{2}{13} \kappa .
\end{gathered}
$$

\section{CHEBYSHEV COLLOCATION METHOD FOR ANALYZING FDDN PENDULUM EQUATION}

Let us now apply the Chebyshev collocation method for analyzing the i.v.p. (3) in the time interval $[0, T]$. To do that, we assume that the solution is given in terms of Chebyshev polynomial

$$
\varphi(t)=\sum_{k=0}^{n} c_{k} T_{k}\left(\frac{2 t-T}{T}\right)
$$

where $T_{k}(t)$ stands for the Chebyshev polynomial of the first kind. We obtain the values of the constants $c_{k}$ by solving the nonlinear algebraic system

$$
\left\{\begin{array}{c}
R\left(t_{k}\right)=0, \\
R(t):=\varphi^{\prime \prime}(t)+2 \beta \varphi^{\prime}(t)+\phi(t) \sin \varphi(t)-F \cos (\Omega t),
\end{array}\right.
$$

where $k=0,1,2, \ldots, n$. 
The collocation points $t_{k}$ are defined as

$$
t_{k}=\frac{1}{2} T\left[1+\cos \left(\frac{4 k+1}{2(n+1)} \pi\right)\right] .
$$

The additional equations are $\varphi(0)=\varphi_{0}$ and $\varphi^{\prime}(0)=\dot{\varphi}_{0}$.

The semi-analytical solution (12) to the i.v.p. (3) could be recovered some special cases. For example:

Case (1) For $\left(\beta, \omega_{2}, F\right)=(0,0,0.1)$, the semi-analytical solution (12) to the forced undamped Duffing oscillator with constant coefficients is plotted against the numerical solutions using both the RK and Chebyshev collocation methods as elucidated in Fig. 1a.

Case (2) For $\left(\beta, \omega_{2}, F\right)=(0,1,0.1)$, the comparison between the semi-analytical solution (12) and both the RK and Chebyshev collocation numerical solutions to the forced undamped Duffing equation with variable coefficients is considered as shown in Fig. 1b.

Case (3) For $\left(\beta, \omega_{2}, F\right)=(0.1,1,0)$, the unforced damped Duffing equation with variable coefficients is recovered and its semi-analytical solution (12) is compared with both the RK and Chebyshev collocation numerical solutions as demonstrated in Fig. 1c.

Case (4) For $\left(\beta, \omega_{2}, F\right)=(0.1,1,0.1)$, the general semianalytical solution (12) to the forced damped parametric driven pendulum i.v.p. (3) is compared with both the RK and Chebyshev collocation numerical solutions as illustrated in Fig. 1d.

Moreover, the maximum distance error for both the semi-analytical solution (12) and the Chebyshev collocation numerical solution with respect to RK numerical solution is estimated for the fourth mentioned cases as seen in Table (I) according to the relation

$$
\left.L_{\infty}\right|_{\text {approx }(\mathrm{Cheb})}=\max _{0 \leq t \leq 40}\left|\varphi_{\text {approx (Cheb) }}-\varphi_{\mathrm{RK}}\right| .
$$

In all cases, it is found that the approximate analytical solutions to the i.v.p. (3) and its related equations are excellent as compared to the numerical solutions. Also, it is observed that the Chebyshev collocation numerical solution is better than the semi-analytical solution (12) for all mentioned cases. However, the semi-analytical solution (12) is more stable than the Chebyshev collocation numerical solution against all relevant physical variables.

\section{CONCLUSIONS}

Some semi-analytical and numerical solutions to the forced damped parametric driven pendulum equation have been reported. This equation has been reduced to the forced damped Duffing equation with variable coefficients in order to find its analytical solution. The approximate analytical solution has been obtained in terms of

TABLE I: A comparison between the maximum distance error of the Chebyshev collocation numerical solution and the semianalytical solution (12) to the i.v.p. (3) with different values of $\left(\beta, \omega_{2}, F, \dot{\varphi}_{0}\right)$.

\begin{tabular}{||c||c||c||}
\hline \hline$\left(\beta, \omega_{2}, F, \dot{\varphi}_{0}\right)$ & $\left.L_{\infty}\right|_{\text {approx }}$ & $\left.L_{\infty}\right|_{\text {Cheb }}$ \\
\hline \hline$(0,0,0.1,0)$ & 0.000214826 & 0.0000883826 \\
\hline \hline$(0,1,0.1,0)$ & 0.00344896 & 0.0000549964 \\
\hline \hline$(0.1,1,0,0.1)$ & 0.000447172 & $4.43839 \times 10^{-6}$ \\
\hline \hline$(0.1,1,0.1,0.1)$ & 0.00326255 & 0.0000770193 \\
\hline \hline
\end{tabular}

the Jacobi elliptic functions. For the numerical analysis, the Chebyshev collocation method has been devoted for solving the equation of motion and some related equations. It was noted that the semi-analytical solution could be recovered some special cases to the nonlinear pendulum oscillations. For instance, in the absence of damping term $(\beta=0)$, the solution to the forced undamped Duffing equation with variable coefficients has been examined. Also, for $\left(\beta, \omega_{2}\right)=(0,0)$, the solution to the forced undamped Duffing equation with constant coefficients has been recovered and discussed. The obtained approximate analytical and Chebyshev collocation numerical solutions have been compared with the RK numerical solutions. Also, the maximum distance error has been estimated for the all solutions. It was found that the semi-analytical solutions give excellent results with high accuracy as compared to the RK numerical solutions. Furthermore, we made a comparison between the Chebyshev collocation numerical solution and the semianalytical solution by estimating the maximum distance errors for them and it was noted that the Chebyshev collocation numerical solution is better than semi-analytical solution. The methods used in this study could be extended to solve many nonlinear equations that control the different cases of pendulum oscillations [25-27]. In addition the obtained results/solutions are useful for investigating several physical problems related to the oscillations in plasma physics, fluid mechanics, field theory, engineering science, solid state physics, quantum mechanics.

\section{REFERENCES}


[1] N. Nayfeth, D.T. Mook., Non-linear oscillations, John Wiley, New York, 1973.

[2] O'Neil T. Physics of Fluids 8, 2255 (1965).

[3] Alvaro H. Salas, S. A. El-Tantawy, and M. R. Alharthi, Phys. Scr. 96 (2021) 104003.

[4] Alvaro H. Salas and S. A.El-Tantawy, "Analytical Solutions of Some Strong Nonlinear Oscillators" DOI: 10.5772/intechopen.97677.

[5] A. Beléndez, C. Pascual, D.I. Méndez, T. Beléndez, and C. Neipp, Revista Brasileira de Ensino de Física, 29, 645 (2007).

[6] P. G. D. Barkham and A. C. Soudack, Int. J. Control 10, 377 (1969).

[7] Noufe H. Aljahdaly and S. A. El-Tantawy, Mathematics 9, 432 (2021).

[8] Alex Elías-Zúñiga, Oscar Martínez-Romero, Daniel Olvera-Trejo, Luis Manuel Palacios-Pineda, Results in Physics 24, 104085 (2021).

[9] M. Turkyilmazoglu, Phys. Scr. 86, 015301 (2012).

[10] H. G. Kadji Enjieu, Orou J. B. Chabi, P. Woafo, Phys. Scr. 77, 025503 (2008).

[11] I. F. Collins, Acta Mechanica 18, 233 (1973).

[12] B. M. Karmakar, J. Eng. Ind. May 100(2), 293 (1978).

[13] J. Zajaczkowski, J. Sound Vib 79, 575 (1981).

[14] S. S. Chang, Eng. Fract. Mech. 18, 887 (1983).

[15] D. Grozev, A. Shivarova, and A. D. Boardman, J. Plasma Phys. 38, 427 (1987).

[16] A. I. J. Manevich, Appl. Math. Mech. 58, 1061 (1994).

[17] R. H. Rand, R. J. Kinsey, and D. L. Mingori, Int. J. Non Linear Mech. 27, 489 (1992).
[18] W. Hu, and D. J. Scheeres, Adv. Astronaut. Sci.105, 839 (2000).

[19] W. Lestari and S. Hanagud, Int. J. Solids Struct. 38, 4741 (2001).

[20] S. Liu, Z. Fu, S. Liu, and Q. Zhao, Phys. Lett. A 289, 69 (2001).

[21] Alvaro H. Salas and Castillo H. Jairo E. Appl. Math. Comput. v.217 fasc.4, 1646 (2010).

[22] Johannessen K. Eur. J. Phys. 35, 035014 (2014).

[23] Yu Guo and Albert C. J. Luo, Bifurcation Dynamics of a Damped Parametric Pendulum, 2019, DOI: 10.2200/S00956ED1V01Y201910MEC022.

[24] S. A. El-Tantawy, Alvaro H. Salas, M. R. Alharthi, Math Probl. Eng. 2021, 6678102 (2021).

[25] J. Ángel Cid, Adv. Nonlinear Anal. 10, 121 (2021).

[26] Gabriela I. Depetri, Felipe A. C. Pereira, Boris Marin, Murilo S. Baptista, and J. C. Sartorelli, Chaos 28, 033103 (2018).

[27] A. Ugulava, Z. Toklikishvili, and S. Chkhaidzea, Chaos 30, 063104 (2020).

\section{Figure caption}

\section{Fig. 1 (Color online)}

A comparison between the semi-analtyical solution (12) and both the Chebyshev collocation and RK numerical solutions to the i.v.p. (3) for different values of $\left(\beta, \omega_{0}, \omega_{2}, \Omega, \gamma, \varepsilon, \varphi_{0}, \dot{\varphi}_{0}, F\right)$. 


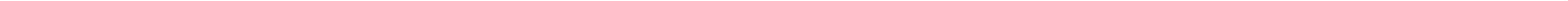

